\section{Endothelial damage and dysfunction in acute graft-versus-host disease}

\author{
Steffen Cordes, ${ }^{1}$ Zeinab Mokhtari, ${ }^{2}$ Maria Bartosova, ${ }^{3}$ Sarah Mertlitz, ${ }^{1}$ Katarina \\ Riesner, ${ }^{1}$ Yu Shi, ${ }^{1}$ Jörg Mengwasser, ${ }^{1,4}$ Martina Kalupa, ${ }^{1}$ Aleixandria McGeary, ${ }^{1}$ \\ Johanna Schleifenbaum, ${ }^{5,6}$ Jens Schrezenmeier, ${ }^{1}$ Lars Bullinger, ${ }^{1}$ Maribel Diaz- \\ Ricart, ${ }^{7}$ Marta Palomo, ${ }^{7,8}$ Enric Carreras, ${ }^{8}$ Gernot Beutel, ${ }^{9}$ Claus Peter Schmitt, \\ ${ }^{3}$ Andreas Beilhack ${ }^{2}$ and Olaf Penack ${ }^{1}$
}

${ }^{1}$ Department of Hematology, Oncology and Tumor Immunology, Charité Universitätsmedizin Berlin, Campus Virchow Clinic, Berlin, Germany; ${ }^{2}$ Department of Medicine II, Würzburg University Hospital, Interdisciplinary Center for Clinical Research (IZKF), Laboratory for Experimental Stem Cell Transplantation, Würzburg, Germany; ${ }^{3}$ Pediatric Nephrology, Center for Pediatric and Adolescent Medicine, University Hospital Heidelberg, Heidelberg, Germany; ${ }^{4}$ Department of Surgery, Charité Universitätsmedizin Berlin, Campus Charité Mitte/Campus Virchow Clinic, Berlin, Germany; ${ }^{5}$ Experimental and Clinical Research Center (ECRC) - a joint cooperation between the Charité Medical Faculty and the Max Delbrück Center for Molecular Medicine (MDC), Berlin, Germany; ${ }^{6}$ Charité Universitätsmedizin Berlin, Institute of Vegetative Physiology, Berlin, Germany; ${ }^{7}$ Department of Hematopathology, Hospital Clinic of Barcelona, Biomedical Diagnosis Centre (CDB), Institute of Biomedical Research August Pi i Sunyer (IDIBAPS), University of Barcelona, Barcelona, Spain; ${ }^{8}$ Josep Carreras Leukemia Research Institute, Hospital Clinic/University of Barcelona Campus, Barcelona, Spain and ${ }^{9}$ Department of Hematology, Hemostasis, Oncology and Stem Cell Transplantation, Hannover Medical School, Hannover, Germany

\section{ABSTRACT}

C linical studies have suggested a potential involvement of endothelial dysfunction and damage in the development and severity of acute graft-versus-host disease (aGvHD). Accordingly, we found an increased percentage of apoptotic caspase 3 positive blood vessels in duodenal and colonic mucosa biopsies of patients with severe aGvHD. In murine experimental aGvHD, we detected severe microstructural endothelial damage and reduced endothelial pericyte coverage accompanied by reduced expression of endothelial tight junction proteins leading to increased endothelial leakage in aGvHD target organs. During intestinal aGvHD, colonic vasculature structurally changed, reflected by increased vessel branching and vessel diameter. As recent data demonstrated an association of endothelium-related factors and steroid refractory aGvHD (SR-aGvHD), we analyzed human biopsies and murine tissues from SR-aGvHD. We found extensive tissue damage but low levels of alloreactive $\mathrm{T}$-cell infiltration in target organs, providing the rationale for T-cell independent SR-aGvHD treatment strategies. Consequently, we tested the endothelium-protective PDE5 inhibitor sildenafil, which reduced apoptosis and improved metabolic activity of endothelial cells in vitro. Accordingly, sildenafil treatment improved survival and reduced target organ damage during experimental SR-aGvHD. Our results demonstrate extensive damage, structural changes, and dysfunction of the vasculature during aGvHD. Therapeutic intervention by endothelium-protecting agents is an attractive approach for SR-aGvHD complementing current anti-inflammatory treatment options.

\section{Introduction}

Allogeneic hematopoietic stem cell transplantation (allo-HSCT) is the only curative treatment option for many patients suffering from hematological malignancies. A major complication of allo-HSCT is acute graft-versus-host disease $(\mathrm{aGvHD})$, an inflammatory condition primarily affecting the skin, liver, and intestines. aGvHD occurs in more than two thirds of patients undergoing allo-HSCT. ${ }^{1}$
Ferrata Storti Foundation

Haematologica 2021

Volume 106(8):2147-2160

\section{Correspondence: \\ OLAF PENACK \\ olaf.penack@charite.de}

Received: March 27, 2020.

Accepted: July 13, 2020.

Pre-published: July 16, 2020.

https://doi.org/10.3324/haematol.2020.253716

(C)2021 Ferrata Storti Foundation

Material published in Haematologica is covered by copyright. All rights are reserved to the Ferrata Storti Foundation. Use of published material is allowed under the following terms and conditions:

https://creativecommons.org/licenses/by-nc/4.0/legalcode. Copies of published material are allowed for personal or internal use. Sharing published material for non-commercial purposes is subject to the following conditions:

https://creativecommons. org//icenses/by-nc/4.0/legalcode, sect. 3. Reproducing and sharing published material for commercial purposes is not allowed without permission in writing from the publisher. 
Steroid treatment is successful in most of these patients ( $75 \%-80 \%)$. However, the $20 \%-25 \%$ of patients who fail initial treatment with steroids (steroid-refractory aGvHD [SR-aGvHD]) have very high mortality., ${ }^{2,3}$ No standard treatment for SR-aGvHD is currently available, and its pathobiology is poorly understood, thereby hindering the development of novel therapeutic approaches.

The endothelium is the first contact for immunological effector cells in the blood and a key regulator in various inflammatory processes. In early complications after alloHSCT such as transplantation-associated-microangiopathy ${ }^{4}$ veno-occlusive disease, ${ }^{5}$ capillary leak syndrome ${ }^{6}$ and diffuse alveolar hemorrhage the endothelium was shown to be relevant. Recent studies also suggest a critical role of the endothelium in aGvHD. Angiogenesis appears to be a very early event during a GvHD, occurring before immune cell infiltration in target organs. ${ }^{8}$ During later phases of aGvHD, endothelial apoptosis has been described in cutaneous aGvHD, ${ }^{9,10}$ Clinical studies demonstrated that soluble markers of endothelial damage such as von Willebrand factor, ${ }^{11}$ thrombomodulin, ${ }^{12-14}$ micro particles released by endothelial cells (EC) ${ }^{15}$ as well as the CD40/CD40 ligand axis, ${ }^{16}$ can be used as biomarkers during aGvHD. Furthermore, factors of endothelial damage were correlated with the mortality rate of patients suffering from SR-aGvHD. ${ }^{12,13,17}$ In addition to endothelial pathology in the microvasculature, arterial vessels were described to suffer from endothelial damage during aGvHD with consequences for their physiological properties. ${ }^{18}$

The characterization of endothelial function during aGvHD and SR-aGvHD is still incomplete and effective pharmacologic strategies aiming at the normalization of endothelial dysfunction to ameliorate aGvHD and SR$\mathrm{aGvHD}$ are lacking.

\section{Methods}

\section{Patient material and histology of human biopsies}

Collection of human samples was approved by the institutional ethics committees of Charité Berlin and Medical University Hannover and was in accordance with the Declaration of Helsinki. From the Charite cohort, we included intestinal biopsies with aGvHD versus no aGvHD after alloHSCT performed between 2007 and 2015. We identified 12 duodenal and 11 colon biopsies from patients with aGvHD grade IIIIV. As a control, we used 19 duodenal and ten colon biopsies from allo-HSCT recipients without histological evidence of aGvHD. ${ }^{19}$ From the Hannover cohort colon biopsies from 11 patients with aGvHD were included. From each patient, biopsies were taken at two time points: at diagnosis of aGvHD and later at diagnosis of SR-aGvHD. Detailed clinical data from both cohorts are given in the Online Supplementary Tables S1, S2, S4 and $S 6$.

\section{Mice and acute graft-versus-host disease experiments}

aGVHD models were used as described previously.,20,21 Control groups (no aGvHD) were transplanted with the same bone marrow (BM) cell numbers and T-cell numbers from syngeneic donors. In order to mimic SR-aGvHD, we used the chemotherapy based murine models $129 \rightarrow \mathrm{B} 6$ major histocompatibility complex (MHC)-matched and B6 $\rightarrow \mathrm{B} 6 \mathrm{D} 2 \mathrm{~F} 1$ (haploidentical) and the radiation based murine model $\mathrm{BALB} / \mathrm{C} \rightarrow \mathrm{B} 6$ MHC-mismatch with conditioning and cell dosages analogue to the models described above. Recipient mice were treated intraperitoneal with $0.5 \mathrm{mg} / \mathrm{kg} /$ day dexamethasone beginning at day+4 after HSCT (Merck, Darmstadt, Germany). ${ }^{22,23}$ We used dexamethasone because of its longer lasting effects compared to methyl-prednisolone or prednisolone enabling once daily dosing in the murine models. The rationale for starting at day +4 is that during this time leukocytes start to infiltrate target organs during aGvHD. ${ }^{8,21,24}$ Clinical scores, weight loss and survival in the B6BDF SR-aGVHD model are given in the Online Supplementary Figure S1.

\section{Histology of murine tissues}

Tissue samples were cryoembedded and scored as previously described. ${ }^{19}$

\section{Evans blue assay}

For assessment of endothelial leakage, Evans blue assay was performed as described in detail elsewhere..$^{25}$

\section{Immunolabeling against VE-cadherin for light sheet fluorescence microscopy}

$25 \mu \mathrm{g} /$ mouse anti-VE-cadherin antibody (Thermo Scientific, eBioBV13-eFluor660) was intravenously (i.v.) injected in mice from B6 $\rightarrow \mathrm{BDF}$ aGvHD model. Mice were sacrificed and perfused with phosphate buffered saline (PBS) followed by $4 \%$ paraformaldehyde in PBS. Sample preparation and imaging of whole organs were performed by light sheet fluorescence microscopy as previously described.$^{26}$ Analysis of vasculature and its segmentation was performed using Imaris 8.1 software (Bitplane, Concord, MA, USA). Branch level was determined by branching point and diameter changes of the vasculature. $^{27}$

\section{Hepatic endothelial cell isolation}

Single cell suspensions were generated via digestion with 2 $\mathrm{mg} / \mathrm{mL}$ collagenase $\mathrm{D}$ and $5 \mu \mathrm{L}$ deoxyribonuclease. Hepatic EC cell fraction was enriched by gradient centrifugation using 30\% histodenz (Sigma Aldrich). For gene expression analysis, the obtained single cell suspension was further enriched for EC (CD11b, CD $\left.45^{\mathrm{dim} /}, \mathrm{CD} 31^{+}\right)$by flow cytometry using a Bio-Rad S3 cell Sorter. EC purity was determined via flow cytometry analysis of ICAM1 $1^{+}$and $\mathrm{CD} 31^{+}$cells.

\section{Statistics}

Survival data were analyzed using the Kaplan-Meier method and compared with the Mantel-Cox log-rank test. For statistical analysis of all other data, Student's $t$-test was used, unless indicated otherwise. Values are presented as mean \pm standard error of the mean (SEM). Values of $P \leq 0.05$ were considered statistically significant. All statistical analyses were performed using GraphPad Prism software (GraphPad Software Inc., La Jolla, CA, USA).

Additional experimental procedures are described in the Online Supplementary Appendix.

\section{Results}

\section{Endothelial apoptosis during severe intestinal acute graft-versus-host disease in human biopsies}

We stained colon and duodenum biopsies from alloHSCT recipients with the apoptotic cell marker caspase 3 (Casp3). We found that Casp3 positive (Casp+) EC were rare events in biopsies of allo-HSCT recipients without 
aGvHD. In contrast, $\mathrm{Casp3}^{+} \mathrm{EC}$ were frequent in intestinal biopsies of patients diagnosed for grade III-IV aGvHD. Figure 1 shows exemplary pictures of colon sections in human allo-HSCT recipients without aGvHD (Figure 1A) versus grade III-IV intestinal aGvHD (Figure 1B). Quantification revealed a significant increase in percentage of $\mathrm{Casp3}^{+}$vessels in duodenal (Figure 1C) and colonic mucosa (Figure 1D) of grade III-IV aGvHD. Patient characteristics and clinical data are given in the Online Supplementary Table S1 (for Figure 1C) and the Online Supplementary Table S2 (for Figure 1D). Our data demonstrate endothelial apoptosis during severe intestinal $\mathrm{aGvHD}$ in human allo-HSCT recipients.

\section{Micro-structural endothelial changes during experimental acute graft-versus-host disease in transmission electron microscopy}

In order to further investigate micro-structural changes of the endothelium we used experimental aGvHD models. We first performed transmission electron microscopy (TEM) of liver (Figure $2 \mathrm{~A}$ to $\mathrm{F}$ ) and colon (Online Supplementary Figure S2A to $F$ ) at day+15 after allo-HSCT. We found that the hepatic sinusoidal endothelium in allo-
HSCT recipients without aGvHD is not affected. The endothelial monolayer as well as the EC-cell contacts were intact (Figure 2A and B), and we observed a normal endothelial monolayer in hepatic sinusoids (Figure 2B). In contrast, the hepatic sinusoidal endothelium during aGvHD was severely damaged with close immune cellEC interactions (Figure 2C). Furthermore, we observed a discontinuous endothelial monolayer at the EC-immune cell contact zone during hepatic aGvHD (Figure 2D). In addition, we found blistering of the endothelial monolayer (Figure 2E) as well as platelet adhesion on the sinusoidal endothelial monolayer (Figure 2E) and in the blistered endothelium (Figure $2 \mathrm{~F}$ ) during hepatic aGvHD. In colonic mucosa, vessels were again normal in allo-HSCT recipients without aGvHD (Online Supplementary Figure $S 2 A$ to $F$ ). The endothelial monolayer was well-structured, smooth and surrounded by pericytes (Online Supplementary Figure S2A) with intact tight junctions (Online Supplementary Figure S2B). In contrast, during intestinal aGvHD the endothelial monolayer was ruffled (Online Supplementary Figure S2C) with perivascular fibrinogen deposits (Online Supplementary Figure S2A, C, D and $F)$. Endothelial cytoplasm was enriched with vesicles

\section{Endothelial apoptosis in human biopsies}

A

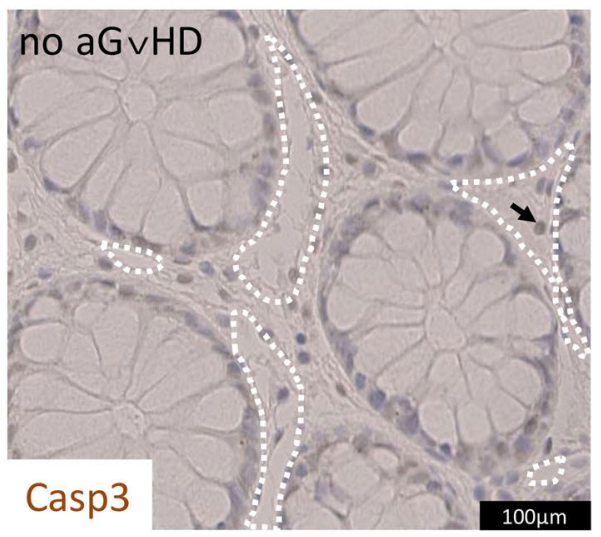

C

Duodenum

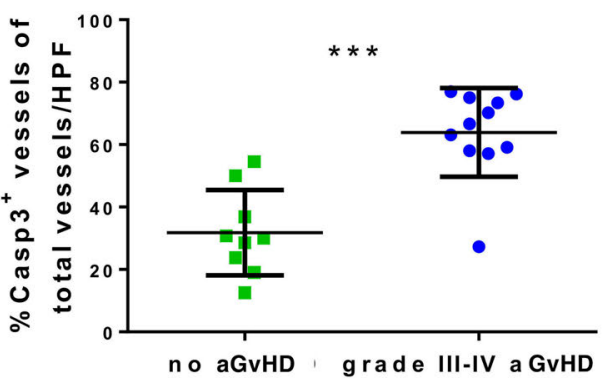

B

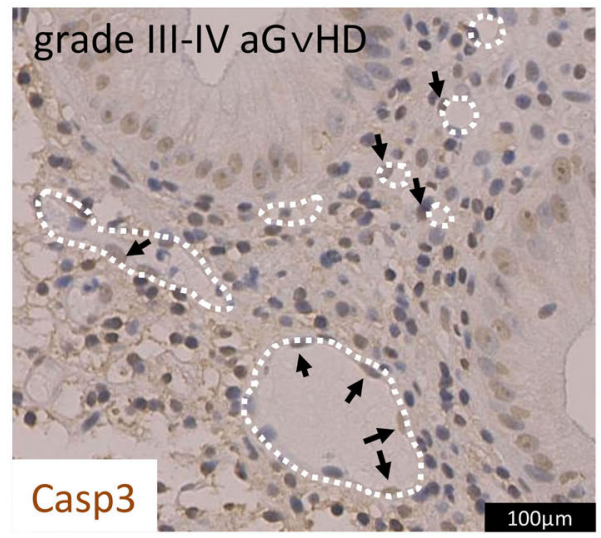

D

Colon

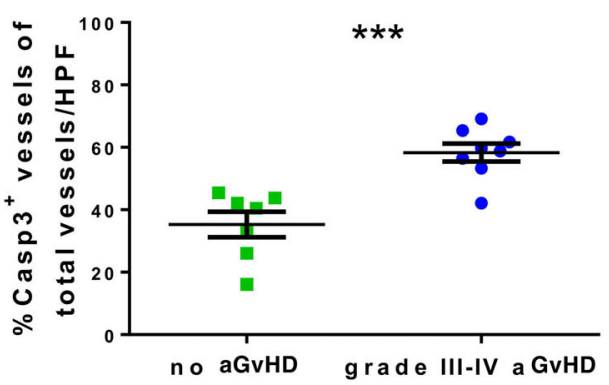

Figure 1. Endothelial damage in human intestinal biopsies. (A) Exemplary picture of a colon biopsy of a patient after allogeneic hematopoietic stem cell transplantation (allo-HSCT) without histologic evidence of acute graft-versus-host disease (aGvHD) and low level of endothelial apoptosis. The white dotted lines indicate vessel lumen and the arrow indicates one apoptotic caspase 3 positive (Casp3 ${ }^{+}$) endothelial cell. (B) Exemplary picture of a colon biopsy of a patient with grade III-IV intestinal aGvHD and increased endothelial apoptosis. The white dotted lines indicate vessel lumen and the arrows indicate apoptotic Casp $3^{+}$endothelial cells. (C) Quantification of Casp3 $^{+}$events in duodenal endothelium of allo-HSCT recipients given in percent of vessels in high-power fields (HPF). (D) Quantification of Casp3 ${ }^{+}$ events in colonic endothelium of allo-HSCT recipients given in percent of vessels in HPF. Percentage of Casp3 ${ }^{+}$vessels was tested for significance by Student's $t$-test $(* * * P<0.001 ; n=7-11$ patients per group). Error bars indicate mean \pm standard error of the mean. 
and the endothelial monolayer was disrupted (Online Supplementary Figure S2D and E). During intestinal aGvHD, clotting was present in the colonic microvasculature as well as convolution of the endothelial monolayer (Online Supplementary Figure S2E). In conclusion, TEM revealed an extensive endothelial damage in target organs during aGvHD.

\section{Pericyte coverage, tight junctions and endothelial leakiness during acute graft-versus-host disease}

As a method to quantify endothelial damage in experimental aGvHD, we analyzed pericyte-coverage of CD $31^{+}$vessels in the colon and liver by fluorescence microscopy. Representative pictures are shown in Figure $3 A$ and $G$. We found reduced pericyte-coverage of vessels in hepatic sinusoids (Figure $3 \mathrm{~B}$ ) and in colonic mucosa (Figure $3 \mathrm{H}$ ) during a GvHD, indicating a damaged endothelial monolayer. In order to address the question whether the endothelial barrier function is altered during aGvHD, we assessed the endothelial expression of the tight junction protein $\mathrm{ZO}-1$ and the intercellular junction protein VE-cadherin in vessels of aGvHD target organs a shown in the representative pictures in Figure $3 \mathrm{C}$ and I. Immunostaining revealed reduced endothelial ZO-1 abundance during aGvHD, which correlates with reduced numbers of intact tight junction (Figure 3D and J). Moreover, colonic microvascular and hepatic sinusoidal endothelium VE-cadherin abundance was reduced (Figure $3 \mathrm{E}$ and $\mathrm{K}$ ).

In order to investigate, if the reduced expression of tight junction and intercellular connection proteins of the endothelium during aGvHD have functional consequences in vivo, we injected Evans blue solution intravenously and analyzed the penetration into the organs. In allo-HSCT recipients with a GvHD endothelial leakage was significantly increased in liver (Figure 3F) and colon (Figure 3L) as compared to HSCT recipients without aGvHD.

Next, we were interested if the detected endothelial changes occurred exclusively in the target organs during
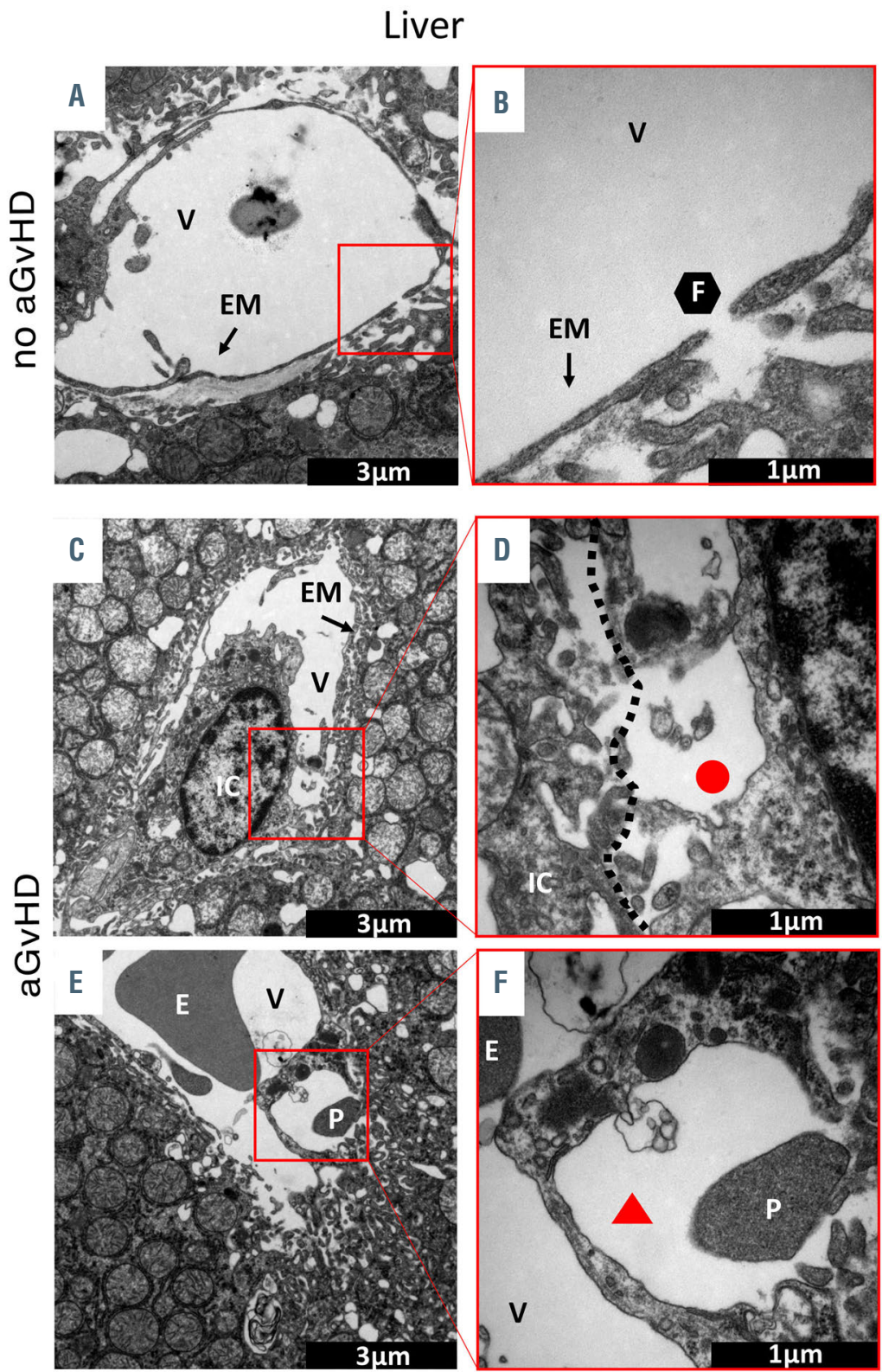

Figure 2. Visualization of acute graft-versus-host disease-associated ultrastructural changes in the liver by transmission electron microscopy. Representative pictures of sections from liver taken at day+15 after experimental hematopoietic stem cell transplantation (HSCT) in the chemotherapy based B6 $\rightarrow$ BDF model. (A and B) Liver sinusoidal endothelial monolayer after syngeneic-HSCT (syn-HSCT) without acute graft-versus-host disease (aGvHD). (A) Normal, fenestrated sinusoidal blood vessel completely covered with endothelial monolayer. (B) Higher magnification of a $100 \mathrm{~nm}$ large fenestration of the endothelium in the liver. (C to-F) Sinusoidal liver endothelial monolayer after allo-HSCT during aGvHD. (C) Liver sinusoida vessel with destroyed and unregularly shaped endothelial monolayer in contact with an immune cell. (D) Higher magnification of contact zone between immune cell and endothelial cell. (E) Blistering of the endothelial monolayer with a platelet in the region of injury. (F) Higher magnification of endothelial blistering. The perivascular space is marked by a red triangle. V: vessel lumen; EM: endothelial monolayer; F: fenestrated endothelium; IC: immune cell; E: erythrocyte; P: platelet; red circle: loss of endothelium; red triangle: endothelial blistering. Control groups (no aGvHD) were transplanted with the same bone marrow cell numbers and T-cell numbers from syngeneic donors. 
A

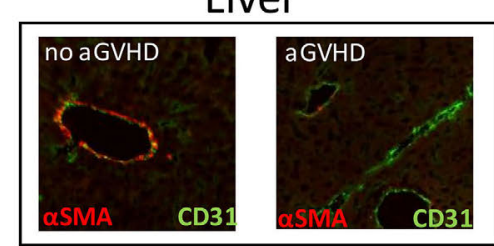

B

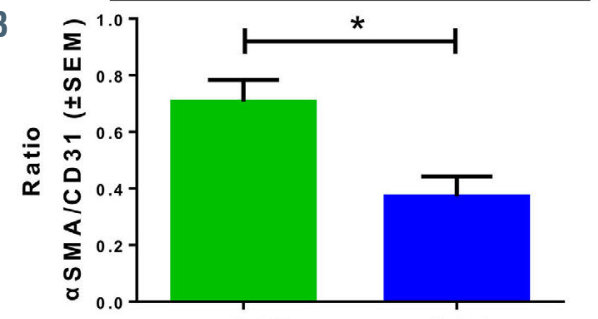

C

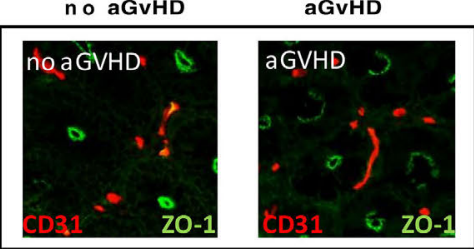

D

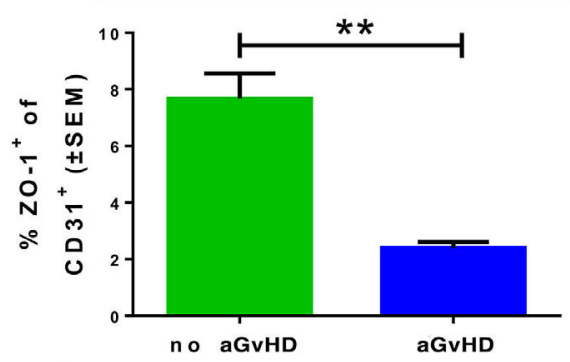

E

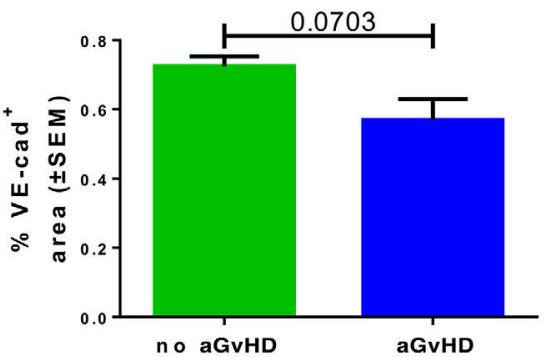

F

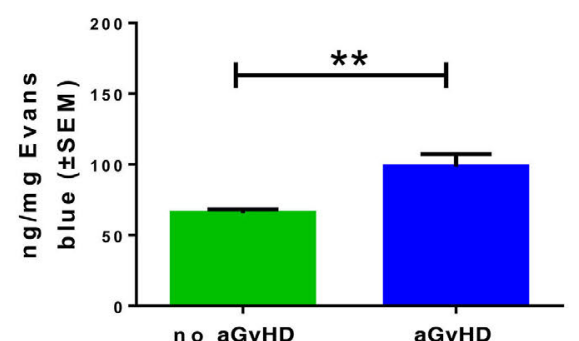

G

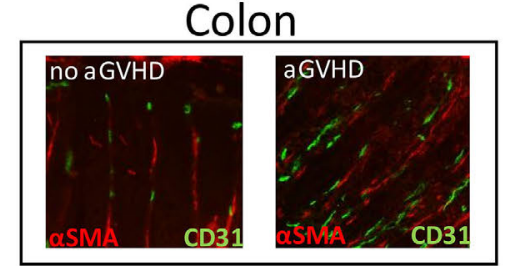

$\mathrm{H}$

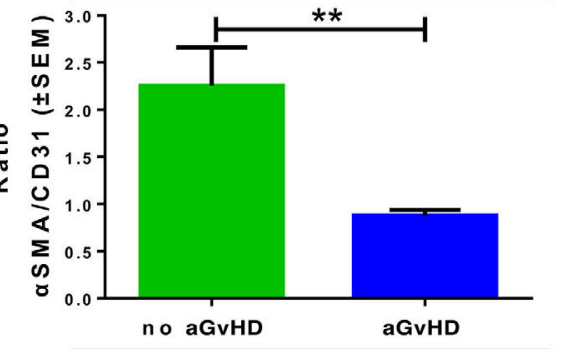

I
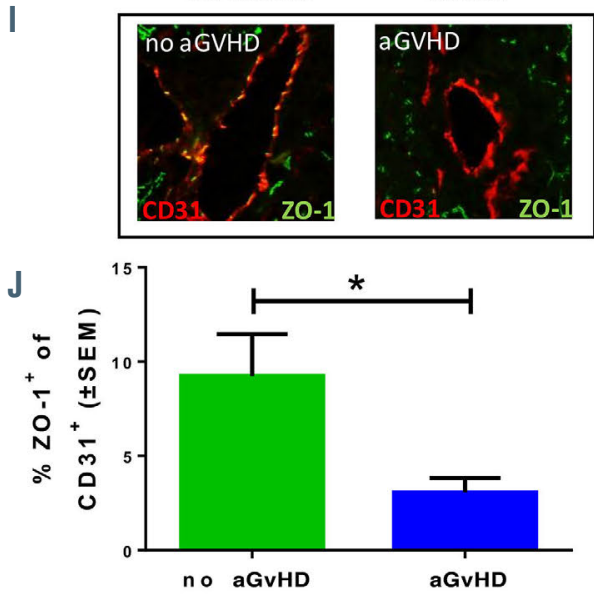

K

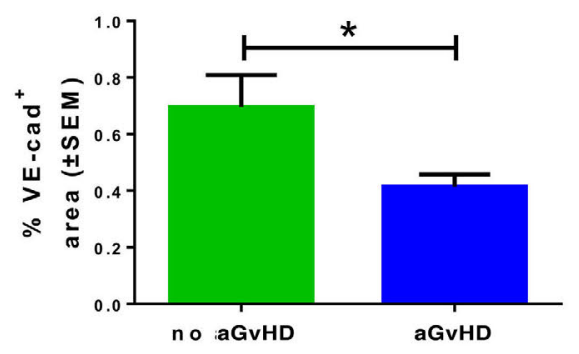

$\mathbf{L}$

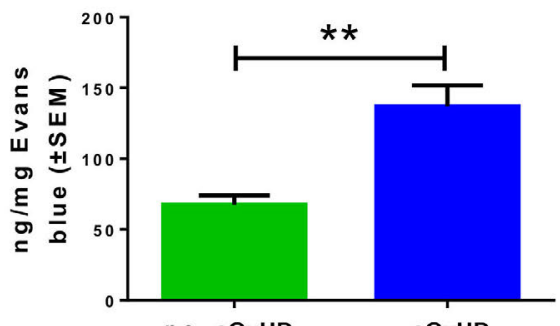

Figure 3. Pericyte coverage, tight junctions and endothelial leakiness during acute graft-versus-host disease. Organs were harvested at day+15 after experimental hematopoietic stem cell transplantation (HSCT) in the chemotherapy based LP/J $\rightarrow B 6$ model. Control groups (no acute graft-versus-host disease [aGvHD]) were transplanted with the same bone marrow cell numbers and T-cell numbers from syngeneic donors. (A, B, and $\mathrm{G}$ to $\mathrm{H}$ ) Quantification of pericyte coverage of vessels. (A and $\mathrm{G})$ Representative pictures of staining for pericyte marker $\alpha$ smooth muscle actin ( $\alpha \mathrm{SMA}$ ) in red and endothelial cell marker CD31 in green. Right organs of animals without aGvHD and left organs of animals suffering from aGvHD. (B and H) Ratio of $\alpha$ SMA positive area and CD31 positive area in (B) liver sinusoidal endothelium and (H) in colonic mucosal vessels in aGvHD versus no aGvHD. (C, D, I and J) Quantification of endothelial tight junction protein expression ZO-1. (C and I) Representative pictures of staining ZO-1 in green and CD31 in red. Right organs of animals without aGvHD and left organs of animals suffering from aGvHD. (D and J) Percentage of ZO$1^{+} \mathrm{CD} 31^{+}$area in (D) liver sinusoidal endothelium and $(\mathrm{J})$ colonic mucosal vessels in aGvHD versus no aGvHD. (E and K) Quantification of endothelial adherence junction protein expression VE-cadherin. Percentage of VE-cadherin positive area in (E) liver sinusoidal endothelium and (K) colonic mucosal vessels in aGvHD versus no aGvHD. $(\mathrm{F}$ and $\mathrm{L}$ ) Measurement of Evans blue extravasation in ng Evans blue per mg at day+15 after experimental allo-HSCT in the chemotherapy based B6 $\rightarrow B D F$ model. ( $\mathrm{B}$ ) Liver and (L) colon of aGvHD versus no aGvHD. Significance was tested with Student's t-test ( $* P<0.05 ; * * P<0.01 ; n=5$ animals per group). All experiments were reproduced in a biological independent experiment and shown are representative results of one experiment. Error bars indicate mean \pm standard error of the mean. 
aGvHD or if this is a systemic effect. We therefore analyzed endothelial leakage in the kidney and skeletal muscle, which are typically not affected in human aGvHD. We have previously confirmed these organs to be unaffected by aGvHD in our models. ${ }^{8,21}$ The Evans blue assay revealed no significant changes of endothelial leakage in these non-target organs during aGvHD (Online Supplementary Figure $3 A$ and $B$ ) suggesting that increased endothelial leakiness predominantly occurs in aGvHD target organs.

Taken together, we found disturbed endothelial tight junctions and intercellular connections leading to increased vascular leakiness in target organs of a GvHD.

\section{Structural changes of vasculature in target organs during aGvHD}

In order to get a better overview of the three-dimensional (3D) changes of the vasculature, we compared $\mathrm{aGvHD}$ and non-GvHD colonic vessel structure and organization with light sheet fluorescence microscopy after in vivo perfusion with an anti-VE-cadherin antibody. Figure $4 \mathrm{~A}$ and $\mathrm{C}$ show normal VE-cadherin staining and vessel branching in a $3 \mathrm{D}$ model in colon at $d+15$ after HSCT without aGvHD. During aGvHD, we found considerably different staining patterns for VE-cadherin (Figure 4B) and increased vessel branching in colon (Figure 4D). Using this $3 \mathrm{D}$ model to quantify vessel branch levels of vasculature, we found significantly increased total vessel branching in colonic vasculature during aGVHD as compared to HSCT recipients without aGvHD (Figure 4E). Accordingly, analysis of branching levels from vessel segments (Figure 4F) and vessel diameter (Figure 4G) revealed a significant increase during intestinal aGvHD. We conclude that aGvHD is associated with structural changes in colonic vasculature.

\section{Dysfunction of the macrovasculature during acute graft-versus-host disease}

As aGvHD is associated with severe signs of microvascular dysfunction, we became interested if a GvHD also influences the physiological functions of macrovasculature as suggested previously. ${ }^{18}$ We examined vessel contraction and relaxation in mouse mesenteric arteries during aGvHD versus no aGVHD after HSCT by myographic measurements. We found that maximum contraction of mesenteric arteries in response to high doses of noradrenaline (NA) and phenylephrine (Phe) was not significantly changed (Online Supplementary Figure $S 4 A$ and $B$ ). However, partial contraction at lower doses of NA and Phe was moderately increased during aGvHD versus no aGvHD (Online Supplementary Figure S4C and D). In addition, fractional relaxation after maximum contraction in response to $\mathrm{ACh}$ was slower in arteries from allo-HSCT recipients with aGvHD (Online Supplementary Figure S4E). Taken together we found increased contraction and reduced relaxation potential of mesenteric arteries during aGvHD indicating systemic hypertension.

\section{Gene expression changes of endothelial cells during acute graft-versus-host disease}

In order to better understand the molecular pathways involved in endothelial dysfunction during aGvHD we performed gene array expression analysis of flow cytometry sorted hepatic EC from HSCT recipients with aGvHD versus without aGvHD at day+15. We found sig- nificant endothelial gene expression changes in complement activation, apoptosis, oxidative damage, IL-1 signaling and cell cycle during aGvHD (Online Supplementary Figures S5, S6 and S7; Online Supplementary Table S3). Furthermore, barrier function and cytoskeleton pathways were differentially regulated, including expression changes of Rho GTPases (Online Supplementary Table 3). We performed interaction analysis of selected endothelium-specific genes (Online Supplementary Figure 7) and found differential expression of Cdh1 and Cdh13 (coding for cadherins), Thbd (coding for thrombomodulin), Vegfc (coding for vascular endothelial growth factor c) and Anpgt1/2 (coding for angiopoietin 1 and 2). In summary, we detected endothelial gene expression changes in different clinically relevant pathways during aGvHD providing possible targets for future therapeutic interventions.

\section{Inflammatory activity and endothelial damage during steroid-refractory acute graft-versus-host disease}

Since recent clinical data has provided evidence on the importance of endothelial pathology specifically in SRaGVHD, ${ }^{12,17,28-31}$ we decided to characterize both inflammatory infiltrates and endothelial damage in this setting. Based on previous data, ${ }^{22}$ we first performed dose-finding studies with steroids in different aGvHD models to establish a protocol with progressive aGvHD despite high dose steroid treatment. Treatment with dexamethasone starting at day+4 after allo-HSCT, showed progressive aGvHD in our models (Online Supplementary Figure S1; Figure 5).

We analyzed vascular pericyte coverage as well as infiltration by $\mathrm{CD}^{+}$donor $\mathrm{T}$ cells in dexamethasonetreated SR-aGvHD versus untreated aGvHD at day +15 after allo-HSCT. We found reduced inflammatory T-cell infiltrates in SR-aGvHD versus untreated aGvHD (Figure $5 \mathrm{~A}$ to $\mathrm{D})$. The predominant remaining cell population in colon during SR-aGvHD were $\mathrm{CD} 4^{+}$and $\mathrm{CD} 8^{+} \mathrm{T}$ cells (Figure $5 \mathrm{C}$ and $\mathrm{D}$ ) as well as $\mathrm{CD} 11 \mathrm{~b}^{+}$and $\mathrm{F} 4 / 80^{+}$myeloid cells (Online Supplementary Figure S8A and B). Endothelial damage as quantified by pericyte coverage reduction in colon vessels was equally severe in SR-aGvHD and untreated aGvHD (Figure 5E). In order to connect the experimental findings to the clinical context of SRaGvHD, we quantified leukocyte infiltration in intestinal biopsies of patients at diagnosis of aGvHD and at later time points of SR-aGvHD in two independent cohorts. Figure panels 5F to J demonstrate significant lower infiltration of $\mathrm{CD}^{4} 5^{+}$leukocytes in colon biopsies as well as duodenum biopsies (Figure 5I). The leukocyte reduction was mainly due to significantly lower infiltration by $\mathrm{CD}^{+} \mathrm{T}$ cells in colon biopsies (Figure $5 \mathrm{~K}, \mathrm{~L}, \mathrm{M}$ and $\mathrm{O}$ ) as well as duodenum biopsies (Figure $5 \mathrm{~N}$ ) during SR$\mathrm{aGvHD}$ versus aGvHD at time of diagnosis. Figure $5 \mathrm{~K}$ gives a typical example of colonic aGvHD at onset with high levels of inflammatory infiltrates by $\mathrm{CD}^{+} \mathrm{T}$ cells and Figure 5L a representative colonic section after steroid treatment and diagnosis for $\mathrm{SR}-\mathrm{aGvHD}$, demonstrating reduced inflammatory infiltrates. In addition, we performed caspase 3 staining and demonstrated a high level of endothelial apoptosis during aGvHD both at diagnosis as well as during SR-aGVHD (Figure 5P). A typical example of high inflammatory activity at aGvHD diagnosis time point and low level of inflammatory activity at diagnosis of SR-GvHD in combination with 
considerable tissue damage in hematoxylin and eosin $(\mathrm{H} \& \mathrm{E})$ staining is given in the Online Supplementary Figure $S 8$, panels $C$ and $D$. Patient characteristics and clinical information is given in the Online Supplementary Tables S4 to $S 6$. Our data indicates that inflammatory activity in intestinal tissues is reduced after steroid treatment, while the endothelial damage is not influenced by steroid treatment during SR-aGvHD.
Endothelial protection by PDE5 inhibition in acute graft-versus-host disease (aGvHD) and steroid-refractory aGvHD

Based on our results demonstrating extensive endothelial damage and reduced inflammatory $\mathrm{T}$-cell infiltration during SR-aGvHD, we hypothesized that pharmacologic protection of the endothelium would have stronger protective effects on SR-aGvHD versus treatment of aGvHD

\section{Colonic vessel structure}
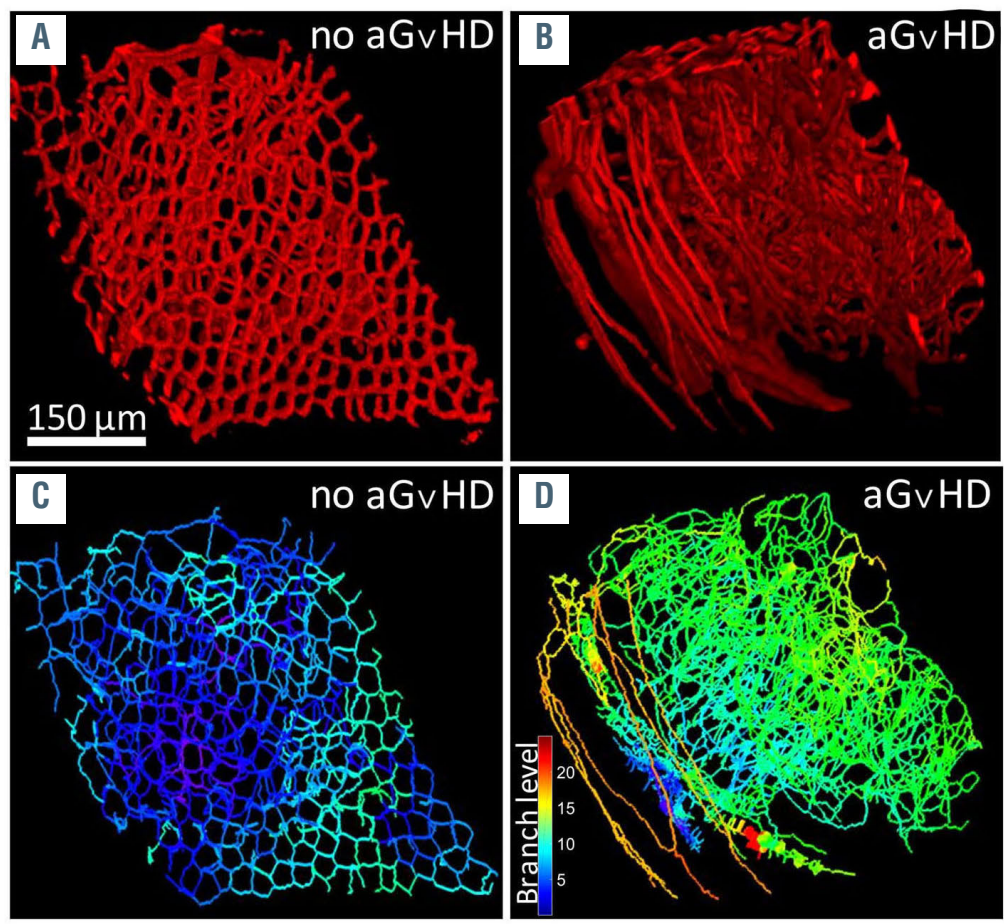

Figure 4. Structural changes of vasculature in target organs during acute graft-versus-host disease by scanning light sheet fluorescence microscopy. We analyzed organs at day+15 after experimental hematopoietic stem cell transplantation (HSCT) in the chemotherapy based $\mathrm{B} 6 \rightarrow \mathrm{BDF}$ model. Contro groups (no aGvHD) were transplanted with the same bone marrow (BM) cell numbers and T-cell numbers from syngeneic donors. (A and B) VE-cadherin signal of vasculature in colon of $(A)$ allogeneic-HSCT (allo-HSCT) recipients without aGvHD and (B) allo-HSCT recipients with aGvHD. (C-D) Computed three-dimensional (3D) model of colonic vasculature with number of branches (blue= low branch levels; red= high branch levels) of (C) allo-HSCT recipients without aGvHD and (D) allo-HSCT recipients with aGvHD. (E to G) Analysis of colonic vasculature parameters. Assessment of (E) total number of branches $(F)$ distribution of vessels branching level and $(G)$ vessel diameter distribution in allo-HSCT recipients with aGvHD versus without aGvHD. Significance of total number of branches was tested by Student's $t$-test $(* * * P<0.001 ; n=3$ animals per group) and significance of vessel branching level was tested by twoway ANOVA with Tukey's multiple comparison test ( $* * * P<0.001 ; n=3$ animals per group). Error bars indicate mean \pm standard error of the mean.
E

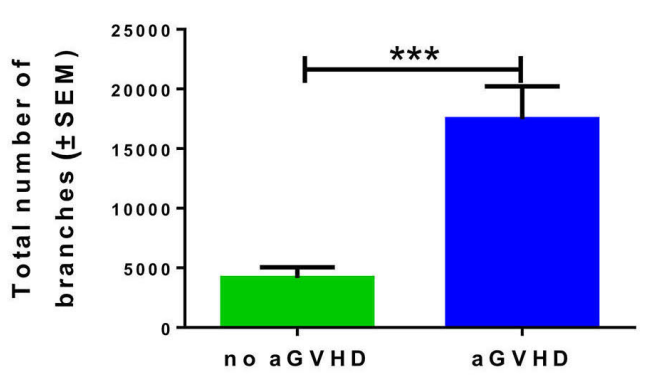

F

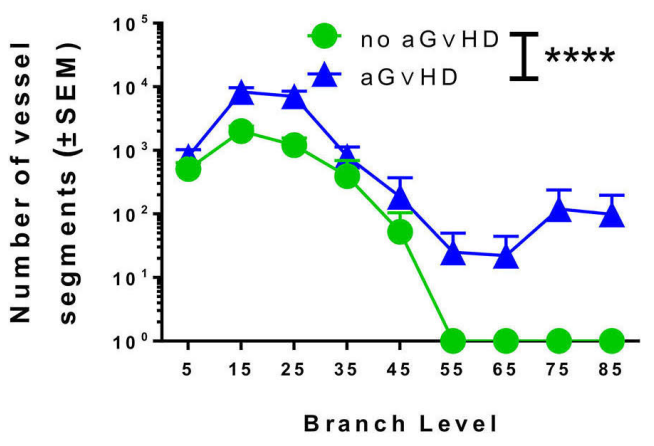

G

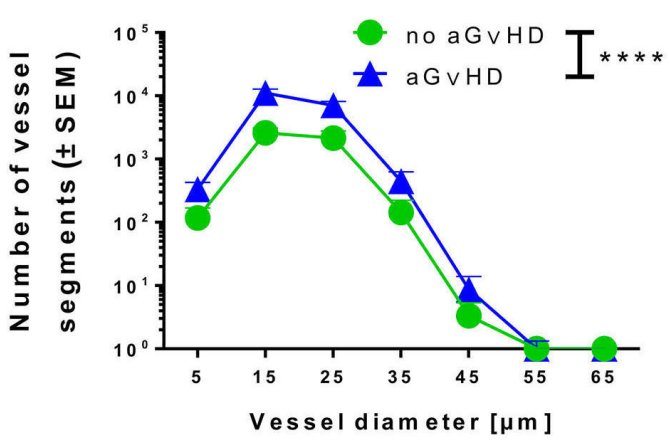



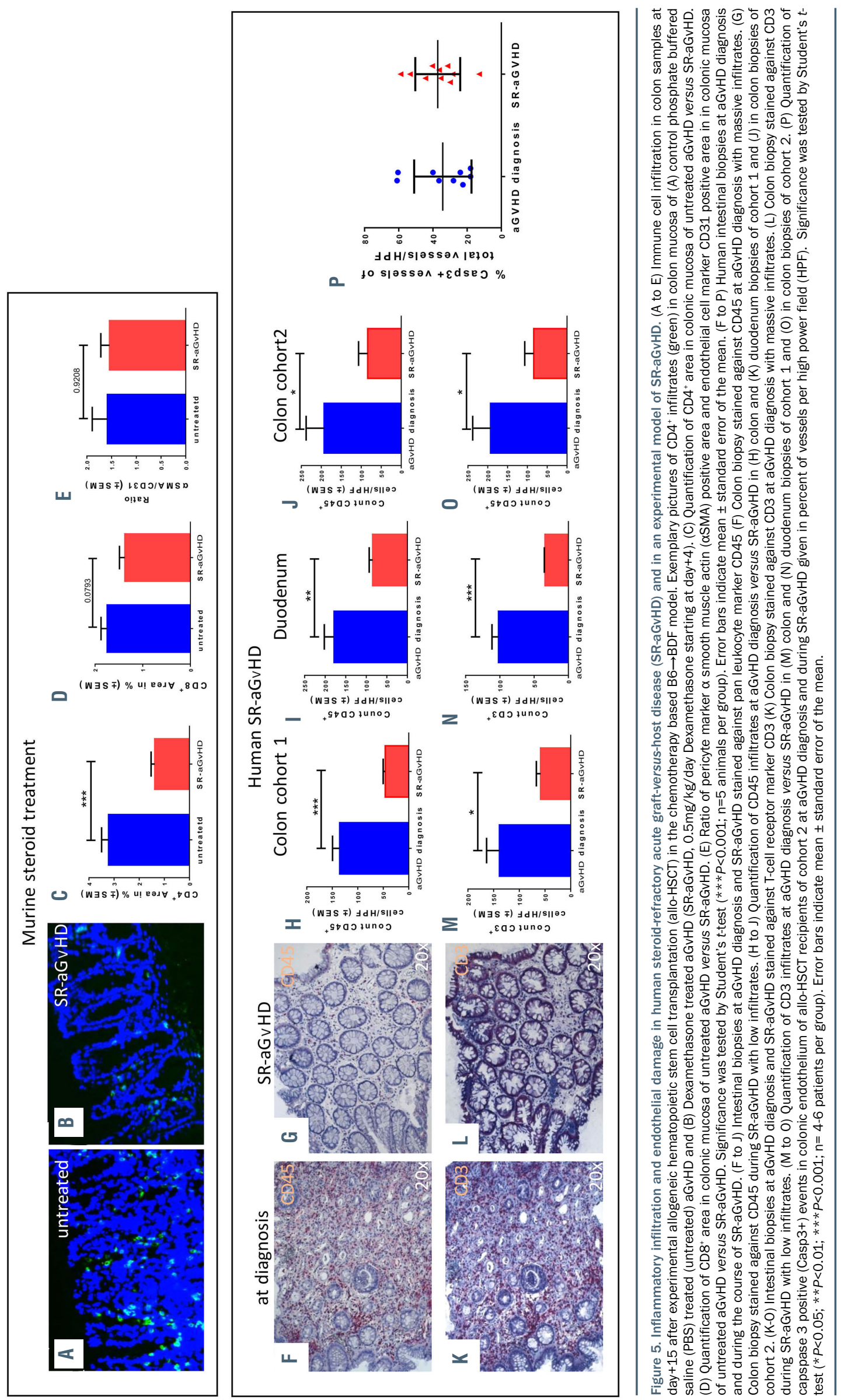


\section{In vitro action of Sildenafil}

A

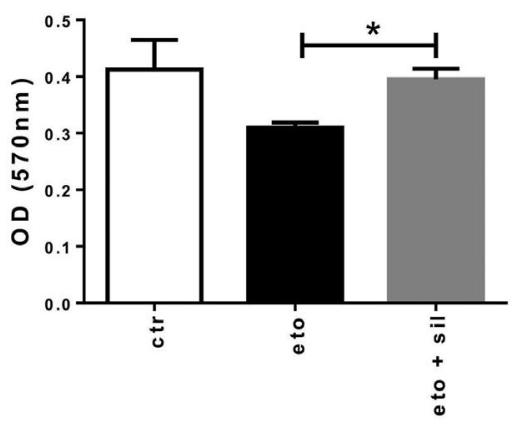

B

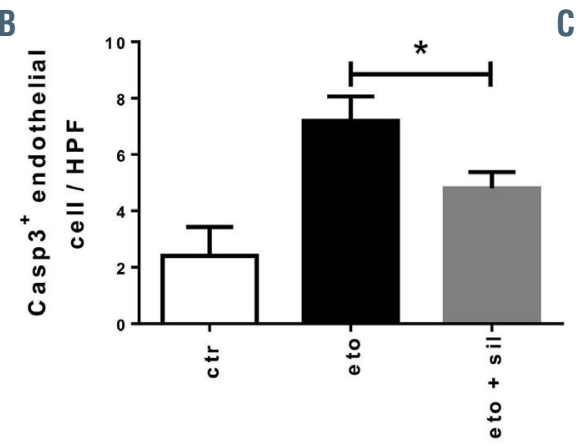

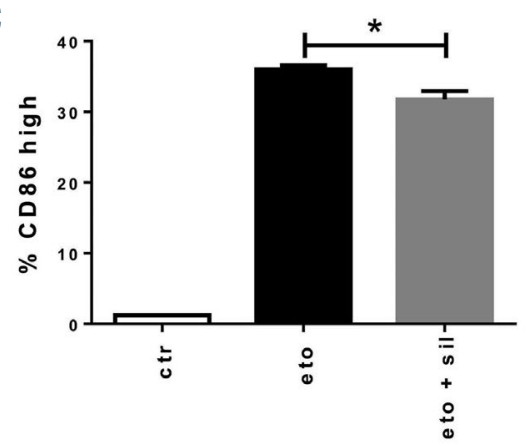

Figure 6. Reduction of endothelial apoptosis and endothelial activation by sildenafil in vitro. Mouse cardiac endothelial cells (MCEC) were incubated with either phosphate buffered saline (PBS)/ 0,1\% dimethylsulfoxide (DMSO) (control [ctr]), $100 \mathrm{~nm}$ etoposide (eto), an inducer of cell death, or with $100 \mathrm{~nm}$ etoposide and $34 \mathrm{~nm}$ sildenafil (eto+sil) for 24 hours before analysis. (A) MTT assay showed higher optical density of eto+sil group versus eto-only group. (B) Staining for apoptotic cell marker caspase 3 (Casp3) showed reduced Casp3 ${ }^{+}$cells per high-power field (HPF) in eto+sil group compared to eto-only group. (C) Flow cytometry analysis of CD86, a costimulatory and endothelial activation marker, showed reduced percentage of endothelial CD86 $6^{\text {high }}$ cells in eto+sil group compared to eto-only group. Significance was tested by Student's $t$-test $\left({ }^{*} P<0.05 ; n=2-3\right.$ experiments with at least triplicates per condition). Error bars indicate mean \pm standard error of the mean.

at its initiation stage. As a first example for such an approach, we used the PDE5 inhibitor sildenafil, which has been demonstrated to normalize endothelial dysfunction in vivo in different settings. ${ }^{32-39}$ First, we tested sildenafil in vitro for EC protection from cytotoxic damage, mediated by etoposide. Sildenafil protected EC from etoposide-induced reduction of endothelial metabolic activity and proliferation (Figure 6A). Sildenafil significantly reduced etoposide-induced endothelial apoptosis, as quantified by $\mathrm{Casp}^{+}$staining (Figure 6B). For co-stimulatory capacity, we checked CD86 $6^{\text {high }}$ expression of EC by flow cytometry. Sildenafil treatment resulted in a significant reduction of etoposide-induced CD86 expression on EC (Figure 6C). Our data demonstrate a protective effect of sildenafil on etoposide-induced endothelial dysfunction in vitro.

We tested the effect of sildenafil in our experimental models of aGvHD (Figure 7A to F) and SR-aGvHD (Figure $7 \mathrm{G}$ to $\mathrm{L}$ ). In the aGvHD model without steroid treatment, we found that sildenafil treatment had no significant effect on survival (Figure 7A). However, sildenafil-treated alloHSCT recipients with aGvHD had significantly lower clinical scores at different time points (Figure 7B) as well as lower histopathological aGvHD scores in the liver (Figure 7C) and colon (Figure 7D) as compared to untreated alloHSCT recipients with aGvHD. In addition, we found a non-significant trend towards reduced costimulatory capacity and antigen presentation potential of hepatic EC under sildenafil treatment (Figure 7E and F). The vascular density (Online Supplementary Figure S9A) as well as the density of lymphatic vessels was not significantly affected by sildenafil treatment (Online Supplementary Figure S9B).

During SR-aGvHD, sildenafil treatment significantly improved survival (Figure 7G). Due to high mortality, clinical scoring was only significant in the early phase (Figure 7H). Histopathological aGvHD scores in the iver (Figure 7I) and colon (Figure 7J) were significantly reduced in sildenafil-treated allo-HSCT recipients with SRaGvHD. Additionally, we found a trend towards reduced co-stimulatory capacity as well as significantly reduced antigen presentation potential of hepatic EC in sildenafiltreated SR-aGvHD, demonstrated by lower MHC class I and II expression (Figure $7 \mathrm{~K}$ ). We confirmed these find- ings in another murine aGvHD model $(129 \rightarrow \mathrm{B} 6$, MHCmatched, Online Supplementary Figure S10). We found non-significant trends towards increased vascular density (Online Supplementary Figure S9C) and lymphatic vascular density in sildenafil-treated SR-GvHD versus controls (Online Supplementary Figure S9D). Next, we analyzed the effect of sildenafil on the endothelium during SR-aGvHD with electron microscopy analyses of liver and colon tissues (Figure $7 \mathrm{M}$ to $\mathrm{P}$ ). We found endothelial damage in the liver and colon in untreated and sildenafil-treated SRaGvHD. However, the sildenafil treated SR-aGvHD group showed reduced endothelial ruptures in liver (Figure $7 \mathrm{~N}$ ) and less prominent fibrinogen deposits in colonic biopsies (Figure 7P).

In order to analyze for effects of sildenafil on T-cell proliferation, we performed in vivo proliferation assays with carboxyfluorescein succinimidyl ester (CFSE) labeled allogeneic $T$ cells. In irradiated mice, sildenafil had no significant effects on in vivo proliferation of allogeneic $\mathrm{T}$ cells (Online Supplementary Figure S11A to C). We next analyzed and quantified the impact on sildenafil on immune cell subsets in peripheral blood during SR-aGvHD and found no significant differences (Online Supplementary Figure $S 11 D$ to $M$ ). Our data suggest that the observed positive effect of sildenafil on SR-aGvHD is predominantly mediated by its effect on EC as opposed to effects on immune cells.

\section{Discussion}

The standardized and well-described aGvHD mice models provide the unique opportunity to experimentally address the role of endothelial dysfunction after HSCT. We found that severe endothelial damage, structural changes of the vasculature and endothelial dysfunction occur during a GvHD. Due to lack of suitable animal models, less detailed data is available for other important endothelium-related complications of allo-HSCT, such as veno-occlusive disease and transplantation-associated microangiopathy. In line with the experimental results, we found that endothelial damage is present in the colon and duodenum of patients with severe aGvHD. This find- 
ing is consistent with a previously published study, demonstrating increased endothelial injury in human skin biopsies during aGvHD. ${ }^{10}$ Additionally, a series of clinical studies have collected supporting evidence on increased endothelial dysfunction during a GvHD, such as high numbers of circulating $\mathrm{EC}^{40-43}$ and elevated serum levels of the endothelial stress markers ST2, von Willebrand factor, angiopoietin 2 and thrombomodulin. ${ }^{31,44-46}$

We have previously shown that aGvHD is associated with increased angiogenesis in target organs. ${ }^{8,47}$ In the current study, we used light sheet fluorescence microscopy to demonstrate that target organ aGvHD is associated with vascular structural changes in terms of higher branching levels and larger diameter of the vasculature. Taking into account the available evidence from our previous studies as well as the experiments from this manuscript, it becomes clear that vascular destruction as well as pathological angiogenesis occur in parallel in the early phase during aGvHD in target organs. These processes finally result in restructuring of the vasculature as visualized and quantified in Figure 4. Interestingly, increased branching levels of vasculature were first reported during pathologi$\mathrm{cal}$ angiogenesis in malignant tumors. ${ }^{48}$ This finding led to the concept of 'vascular normalization' to improve antitumor therapies. ${ }^{49}$ However, the significance of pathological vascular organization with increased branching level for the pathophysiology of a GvHD and possible therapeutic implications remains to be determined.

We found that the expression of tight junction- and adherence junction proteins were reduced during aGVHD, leading to increased vascular permeability in a GvHD target organs after allo-HSCT. The role of tight junction- and adherence junction proteins for vascular barrier maintenance ${ }^{50-52}$ as well as leukocyte transmigra$\operatorname{tion}^{53,54}$ has been described previously. Additionally, the promotion of the adherence junction protein VE-cadherin in tumor vasculature increased T-cell infiltration into the tumor. $^{55}$ These findings may open a new window for

\section{Murine aGvHD}

A

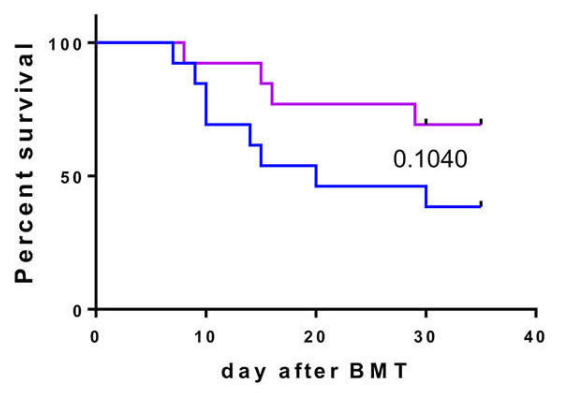

C

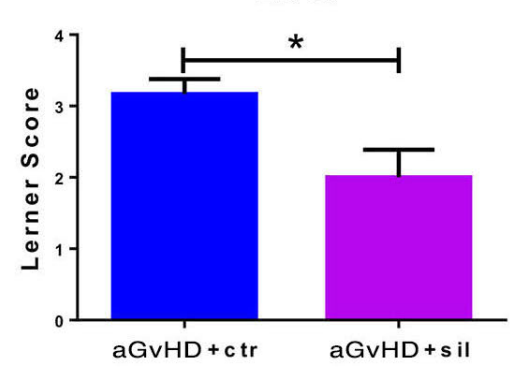

E

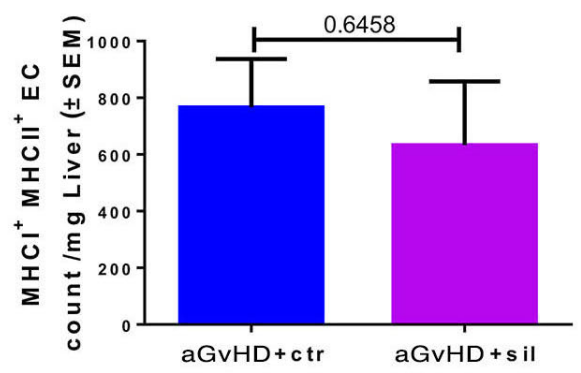

B

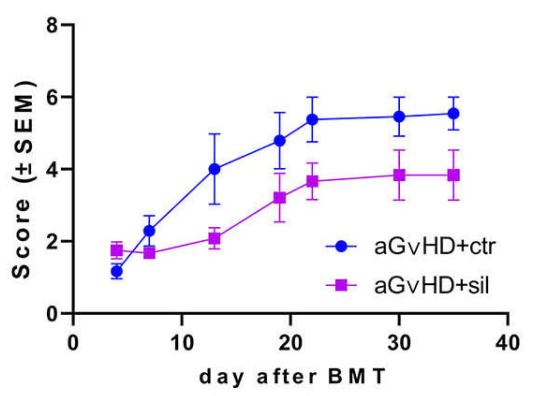

D

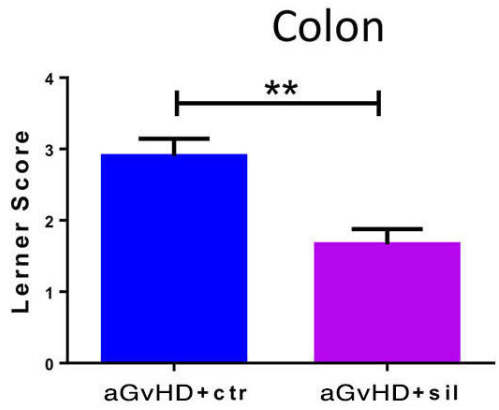

F

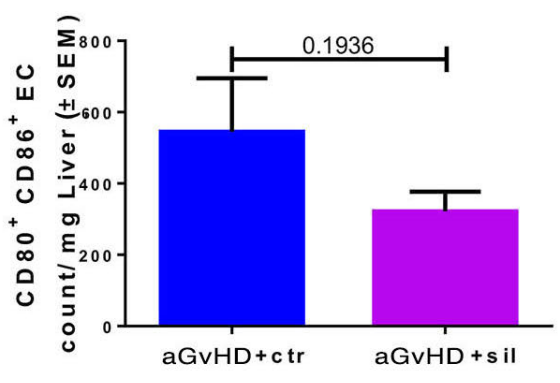

Figure 7. In vivo treatment of acute graft-versus-host disease (aGvHD) and steroid-refractory-aGvHD with sildenafil. (A to G) Treatment of aGvHD with $10 \mathrm{mg} / \mathrm{kg} / \mathrm{d}$ sildenafil after experimental allogeneic hematopoietic stem cell transplantation (allo-HSCT) at day+15 after experimental allo-HSCT in the radiation based $B 6 \rightarrow B A L B / C$ model. (A) Survival analysis and (B) clinical aGvHD manifestations of sildenafil (sil) treated allo-HSCT recipients with aGvHD versus control substance phosphate buffered saline/dimethylsulfoxide (PBS/DMSO, control [ctr]) treated allo-HSCT recipients with aGvHD. Histopathological assessment of aGvHD manifestations in (C) liver and (D) colon in sildenafil versus control substance treated allo-HSCT recipients at day+15. Flow cytometry quantification of (E) major histocompatibility complex I (MHCl) and $\mathrm{MHCll}$ expression and $(\mathrm{F}) \mathrm{CD} 80$ and $\mathrm{CD} 86$ expression of isolated liver sinusoidal endothelial cells of sildenafil versus control substance treated allo-HSCT recipients at day+15. (G to L) Treatment with $10 \mathrm{mg} / \mathrm{kg} / \mathrm{d}$ sildenafil in a murine model of SR-aGvHD at day+15 after experimental allo-HSCT in the radiation based $\mathrm{B} 6 \rightarrow \mathrm{BALB} / \mathrm{C}$ model. (continued on next page.) 
Murine SR-aG vHD

G
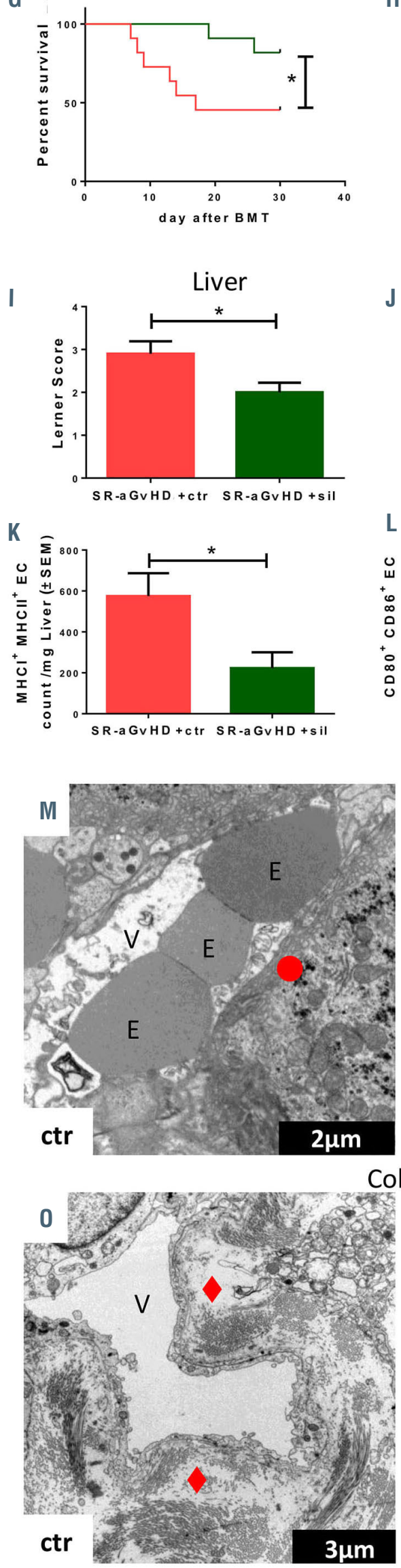

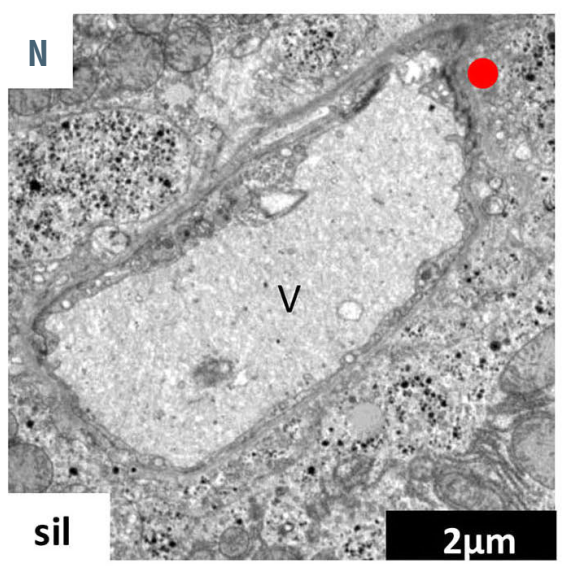

$\mathrm{H}$

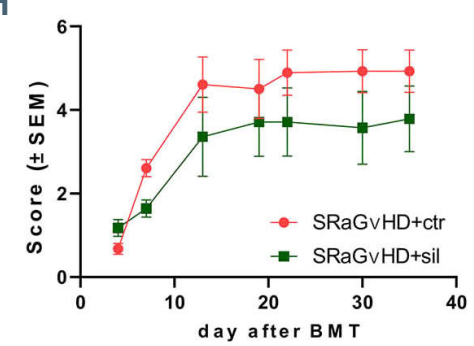

Colon
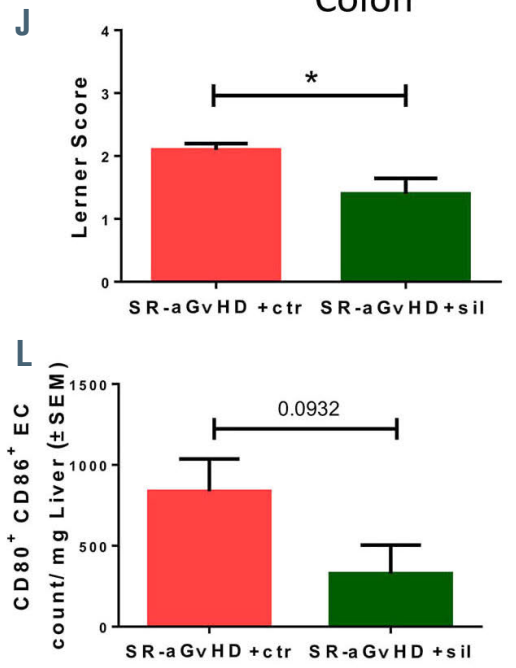

Colon

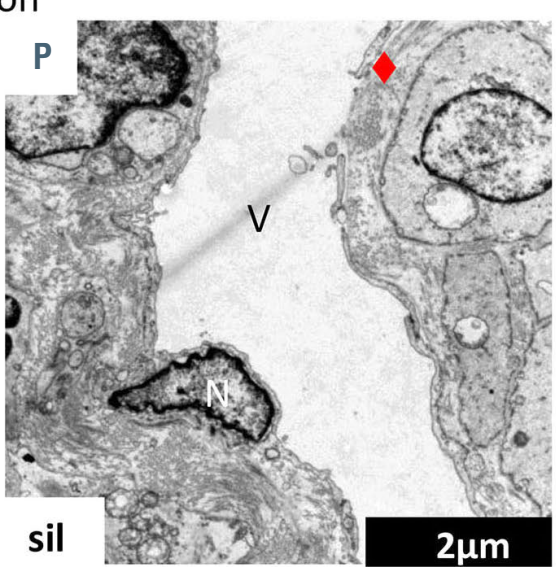

Figure 7. (G) Survival analysis and $(\mathrm{H})$ clinical aGvHD manifestations of sildenafil (SR-aGvHD+sil) versus control substance treated (SR-aGvHD+ctr) SR-aGvHD. Histopathological assessment of aGvHD severity in (I) liver and $(J)$ colon in SRaGvHD+sil and SR-aGvHD+ctr at day+15 after allo-HSCT. Flow cytometry quantification of $(\mathrm{K}) \mathrm{MHCl}$ and $\mathrm{MHCll}$ expression and (L) CD80 and CD86 expression of isolated liver sinusoidal endothelial cells of SR-aGvHD+sil and SR-aGvHD+ctr at day+15 after allo-HSCT. Significance was tested by Kaplan-Meier method and compared with the Mantel-Cox log-rank test ( $* P<0.05 ; n=8-10$ animals per group) and Student's $t$-test $(* P<0.05 ; * * P<0.01$; $\mathrm{n}=6$ animals per group). Error bars indicate mean \pm standard error of the mean. Survival data was pooled from two experiments. All experiments were reproduced in a biological independent experiment and shown are representative results of one experiment. ( $\mathrm{M}$ to $\mathrm{P}$ ) Visualization of SR-aGvHD and sildenafil treated SRaGvHD associated ultrastructural changes in the liver and the colon by transmission electron microscopy. Shown are typical pictures of sections from liver and colon taken at day+15 after experimental allo-HSCT in the chemotherapy based 129 $\rightarrow$ B6 model. ( $M$ and $N$ ) Sinusoidal liver endothelial monolayer during SR-aGvHD and sildeanfil (sil) treated SR-aGvHD. (M) Liver sinusoidal vessel of untreated SR-aGvHD with destroyed and unregularly shaped endothelial monolayer, marked by a red circle. (N) Liver sinusoidal vessel of Sildenafil treated SR-aGvHD with small ruptures of the endothelial monolayer, marked by a red circle. ( $\mathrm{O}$ and $\mathrm{P}$ ) Colonic mucosa endothelium during SR-aGvHD and sildeanfil treated SR-aGvHD, (O) The vessel of untreated SR-aGvHD is surrounded by massive perivascular fibrinogen deposits marked by a red rhombus. (P) Occasionally little perivascular fibrinogen deposits, marked by a red rhombus, could be detected in sildenafil treated SRaGvHD. V: vessel lumen; E: erythrocyte; N: nucleus; red rhombus: perivascular fibrinogen deposits; red circle: loss of endothelium; red trapezoid: endothelial convolution). 
aGvHD therapies aiming at reducing vascular permeability and stabilization of vascular tight-junctions as well as adherence-junctions. Vestweber and colleagues have generated mice with stabilized endothelial junctions; these mice had strongly reduced neutrophil and lymphocyte recruitment into inflamed tissues. ${ }^{56}$ Examples for a therapeutic approach derive from the field of sepsis where reduction of vascular permeability with the $\alpha V \beta 3$ antagonist cilengitide ${ }^{57}$ or with an antibody binding to angiopoietin 2 and TIE2 ${ }^{58}$ which led to substantial biological benefits.

Recent clinical studies suggest a role of endothelial pathology particularly in SR-aGvHD ${ }^{13,14,17,28,29,31}$ and a high medical need for development of respective therapies. We now demonstrate in two independent clinical cohorts as well as in experimental models that inflammatory infiltration in target organs is considerably reduced compared to $\mathrm{aGvHD}$ at diagnosis. Consequently, we found that dexamethasone treatment did not significantly reduce the severity of endothelial damage during SR-aGvHD. Pavan Reddy's group recently published the results of extensive experiments in murine models of SR-GvHD. In line with our findings, their results point towards the existence of T-cell independent mechanisms in the pathophysiology of SR-GvHD. ${ }^{23}$ This may explain the historically disappointing clinical results of immunosuppressive treatments for SR-aGvHD. The low inflammatory status in connection with considerable endothelial pathology provides a rationale for therapies protecting the endothelium during SR-aGvHD. Based on previous encouraging data on normalization of endothelial dysfunction, ${ }^{32-39}$ we used the PDE5 inhibitor sildenafil as a first attempt to treat SRaGvHD by an 'endothelium-protective' approach. Sildenafil treatment ameliorated aGvHD and the effect was more pronounced in case of SR-aGvHD compared with previously untreated a GvHD. The latter observation may be explained by the high level of tissue inflammation in non-glucocorticoid treated aGvHD mice superimposing the beneficial effects of sildenafil on EC. However, we cannot exclude the possibility of a synergistic effect of sildenafil with steroids in our models.

The direct mechanisms of sildenafil treatment on the course of SR-aGvHD remain unclear. However, improvement of vascular barrier function and reduction of EC apoptosis probably contribute to the beneficial effects of sildenafil. During aGvHD we found increased vascular permeability in target organs and alterations of Rho GTPases, who are critical regulators of endothelial barrier function. ${ }^{59}$ Sildenafil-mediated stabilization of endothelial barrier function ${ }^{60,61}$ as well as impact of sildenafil on regulation of Rho GTPases ${ }^{62}$ have been described previously. We found that apoptosis and expression of relevant genes, such as nitric oxide synthase (Nos3) and BCL-2, was increased in target organ EC during aGvHD. Sildenafil has been shown to have cyto-protective effects by regulation of nitric oxide synthase 3 signaling and BCL-2 expression. ${ }^{63}$ In addition, sildenafil may have positive effects by stabilizing vessel surrounding pericytes and thereby vascular integrity: PDE5 targets cGMP, which has been demonstrated to inhibit the proliferation of smooth muscle cells by causing a delay in $G_{1} / S$ transition in the cell cycle. ${ }^{64}$ Interestingly, we detected differentially regulated cell cycle genes in EC during aGvHD. Of note, we found that sildenafil treatment led to reduction of aGvHD-associated expression of co-stimulatory molecules on target organ EC. However, our finding that sildenafil had no significant effects on in vivo proliferation of allogeneic donor $T$ cells in irradiated hosts argue against the biologic significance of the observed impact of sildenafil on EC co-stimulatory molecule expression during aGvHD.

In conclusion, our results demonstrate extensive damage, structural changes, and dysfunction of the vasculature during a GvHD, and our findings suggest a novel concept of therapeutic intervention by endothelium-protecting agents as an attractive treatment approach for SRaGvHD. A likely future perspective is to test endothelium-targeting approaches complementing treatment options with proven efficacy in SR-aGvHD, such as JAK2 inhibition. ${ }^{65}$

\section{Disclosures}

No conflicts of interest to disclose.

\section{Contributions}

$S C, A B, E C, M D-R, J M, L B, C P S$ and $O P$ designed the study; SC, ZM, MB, YS, SM, KR, JM, MK, AM, JS, MP and $G B$ performed experiments and analyzed results; $S C$ and $O P$ wrote the manuscript. All authors read, edited and approved the manuscript.

\section{Acknowledgments}

This work was supported by the Jose Carreras LeukämieStiftung (11R2016, 03R 2019), Deutsche Krebshilfe (70113519), Deutsche Forschungsgemeinschaft (PE 1450/7-1), Monika-Kutzner-Stiftung, and Wilhelm-Sander-Stiftung (2014.150.1).

\section{Funding}

$M B$ was funded by the European Training and Research in Peritoneal Dialysis Program, funded by the European Union within the Marie Curie Scheme (287813). AB received funding from the German Research Foundation (DFG), collaborative research center TRR221 (B11, ZO2) and ZM was funded by the $D F G$ collaborative research center TRR225 (B08).

\section{References}

1. Garnett C, Apperley JF, Pavlu J. Treatment and management of graft-versus-host disease: improving response and survival. Ther Adv Hematol. 2013;4(6):366-378.

2. Deeg HJ. How I treat refractory acute GVHD. Blood. 2007;109(10):4119-4126.

3. Westin JR, Saliba RM, De Lima M, et al. Steroid-refractory acute GVHD: predictors and outcomes. Adv Hematol. 2011;2011: 601953.

4. Gloude NJ, Khandelwal P, Luebbering N, et al. Circulating dsDNA, endothelial injury, and complement activation in thrombotic microangiopathy and GVHD. Blood. 2017;130(10):1259-1266.

5. Fan CO, Crawford JM. Sinusoidal obstruction syndrome (hepatic veno-occlusive disease). J Clin Exp Hepatol. 2014;4(4):332346.

6.Xie Z, Ghosh CC, Patel R, et al. Vascular endothelial hyperpermeability induces the clinical symptoms of Clarkson disease (the systemic capillary leak syndrome). Blood. 2012;119(18):4321-4332.
7. Ueda N, Chihara D, Kohno A, et al. Predictive value of circulating angiopoietin2 for endothelial damage-related complications in allogeneic hematopoietic stem cell transplantation. Biol Blood Marrow Transplant. 2014;20(9):1335-1340.

8. Riesner K, Shi Y, Jacobi A, et al. Initiation of acute graft-versus-host disease by angiogenesis. Blood. 2017;129(14):2021-2032.

9. Murata H, Janin A, Leboeuf C, et al. Donorderived cells and human graft-versus-host disease of the skin. Blood. 2007;109(6): 2663-2665. 
10. Dumler JS, Beschorner WE, Farmer ER, Di Gennaro KA, Saral R, Santos GW. Endothelial-cell injury in cutaneous acute graft-versus-host disease. Am J Pathol. 1989;135(6):1097-1103.

11. Mir E, Palomo M, Rovira $M$, et al. Endothelial damage is aggravated in acute GvHD and could predict its development. Bone Marrow Transplant. 2017;52(9):13171325.

12. Rachakonda SP, Penack O, Dietrich S, et al. Single-nucleotide polymorphisms within the thrombomodulin gene (THBD) predict mortality in patients with graft-versus-host disease. J Clin Oncol. 2014;32(30): 34213427.

13. Dietrich S, Falk CS, Benner A, et al. Endothelial vulnerability and endothelial damage are associated with risk of graftversus-host disease and response to steroid treatment. Biol Blood Marrow Transplant. 2013;19(1):22-27.

14. Andrulis M, Dietrich S, Longerich T, et al. Loss of endothelial thrombomodulin predicts response to steroid therapy and survival in acute intestinal graft-versus-host disease. Haematologica. 2012:97(11):16741677.

15. Pihusch V, Rank A, Steber R, et al. Endothelial cell-derived microparticles in allogeneic hematopoietic stem cell recipients. Transplantation. 2006;81(10):14051409

16. Rachakonda SP, Dai H, Penack O, et al. Single nucleotide polymorphisms in CD40L predict endothelial complications and mortality after allogeneic stem-cell transplantation. J Clin Oncol. 2018;36(8): 789-800

17. Luft T, Benner A, Jodele S, et al. EASIX in patients with acute graft-versus-host disease: a retrospective cohort analysis. Lancet Haematol. 2017;4(9):e414-e423.

18. Schmid PM, Bouazzaoui A, Doser K, et al. Endothelial dysfunction and altered mechanical and structural properties of resistance arteries in a murine model of graft-versus-host disease. Biol Blood Marrow Transplant. 2014;20(10):14931500.

19. Lerner KG, Kao GF, Storb R, Buckner CD, Clift RA, Thomas ED. Histopathology of graft-vs.-host reaction (GvHR) in human recipients of marrow from HL-A-matched sibling donors. Transplant Proc. 1974:6(4):367-371

20. Mertlitz S, Shi Y, Kalupa M, et al. Lymphangiogenesis is a feature of acute GVHD, and VEGFR-3 inhibition protects against experimental GVHD. Blood. 2017;129(13):1865-1875

21. Riesner K, Kalupa M, Shi Y, Elezkurtaj S, Penack O. A preclinical acute GVHD mouse model based on chemotherapy conditioning and MHC-matched transplantation. Bone Marrow Transplant. 2016;51(3):410-417.

22. Bouazzaoui A, Spacenko E, Mueller G, et al. Steroid treatment alters adhesion molecule and chemokine expression in experimental acute graft-vs.-host disease of the intestinal tract. Exp Hematol. 2011;39(2): 238-249 e231.

23. Toubai T, Rossi C, Tawara I, et al. Murine models of steroid refractory graft-versushost disease. Sci Rep. 2018;8(1):12475

24. Beilhack A, Schulz S, Baker J, et al. In vivo analyses of early events in acute graft-versus-host disease reveal sequential infiltration of T-cell subsets. Blood. 2005;106(3) 1113-1122.
25. Radu M, Chernoff J. An in vivo assay to test blood vessel permeability. J Vis Exp. 2013(73): e50062.

26. Brede C, Friedrich M, Jordan-Garrote AL, et al. Mapping immune processes in intact tissues at cellular resolution. J Clin Invest. 2012;122(12):4439-4446

27. Wertheimer T, Velardi E, Tsai J, et al. Production of BMP4 by endothelial cells is crucial for endogenous thymic regeneration. Sci Immunol. 2018;3(19):eaal2736.

28. Dietrich S, Okun JG, Schmidt K, et al. High pre-transplant serum nitrate levels predict risk of acute steroid-refractory graft-versushost disease in the absence of statin therapy. Haematologica. 2014;99(3):541-547.

29. Luft T, Dietrich S, Falk C, et al. Steroidrefractory GVHD: T-cell attack within a vulnerable endothelial system. Blood. 2011;118(6):1685-1692.

30. Rotz SJ, Dandoy CE, Davies SM. ST2 and Endothelial injury as a link between GVHD and microangiopathy. $\mathrm{N}$ Engl J Med. 2017:376(12):1189-1190

31. Vander Lugt MT, Braun TM, Hanash S, et al. ST2 as a marker for risk of therapy-resistant graft-versus-host disease and death. $\mathrm{N}$ Engl J Med. 2013;369(6):529-539.

32. Balarini CM, Leal MA, Gomes IB, et al. Sildenafil restores endothelial function in the apolipoprotein E knockout mouse. J Transl Med. 2013;11:3.

33. Bivalacqua TJ, Sussan TE, Gebska MA, et al. Sildenafil inhibits superoxide formation and prevents endothelial dysfunction in a mouse model of secondhand smoke induced erectile dysfunction. J Urol. 2009;181(2):899-906

34. Aksu V, Yuksel V, Chousein S, et al. The effects of sildenafil and n-acetylcysteine on ischemia and reperfusion injury in gastrocnemius muscle and femoral artery endothelium. Vascular. 2015;23(1):21-30.

35. Valatsou A, Briasoulis A, Vogiatzi G, et al. Beneficial effects of sildenafil on tissue perfusion and inflammation in a murine model of limb ischemia and atherosclerosis. Curr Vasc Pharmacol. 2017;15(3):282-287.

36. Fahning BM, Dias AT, Oliveira JP, et al. Sildenafil improves vascular endothelial structure and function in renovascular hypertension. Curr Pharm Biotechnol. 2015;16(9):823-831.

37. McLaughlin K, Lytvyn Y, Luca MC, Liuni A, Gori T, Parker JD. Repeated daily dosing with sildenafil provides sustained protection from endothelial dysfunction caused by ischemia and reperfusion: a human in vivo study. Am J Physiol Heart Circ Physiol. 2014;307(6):H888-894.

38. Yin J, Kukucka M, Hoffmann J, et al. Sildenafil preserves lung endothelial function and prevents pulmonary vascular remodeling in a rat model of diastolic heart failure. Circ Heart Fail. 2011:4(2):198-206.

39. Yaguas K, Bautista R, Quiroz Y, et al. Chronic sildenafil treatment corrects endothelial dysfunction and improves hypertension. Am J Nephrol. 2010;31(4): 283-291.

40. Almici C, Skert C, Bruno B, et al. Circulating endothelial cell count: a reliable marker of endothelial damage in patients undergoing hematopoietic stem cell transplantation. Bone Marrow Transplant. 2017;52(12):1637-1642.

41. Woywodt A, Scheer J, Hambach L, et al. Circulating endothelial cells as a marker of endothelial damage in allogeneic hematopoietic stem cell transplantation. Blood. 2004;103(9):3603-3605.
42. Medinger M, Heim D, Gerull S, et al. Increase of endothelial progenitor cells in acute graft-versus-host disease after allogeneic haematopoietic stem cell transplantation for acute myeloid leukaemia. Leuk Res. 2016:47:22-25.

43. Yan Z, Zeng L, Jia L, Xu S, Ding S. Increased numbers of circulating ECs are associated with systemic GVHD. Int J Lab Hematol. 2011;33(5):507-515.

44. Tatekawa S, Kohno A, Ozeki K, et al. A novel diagnostic and prognostic biomarker panel for endothelial cell damage-related complications in allogeneic transplantation. Biol Blood Marrow Transplant. 2016;22(9):1573-1581.

45. Lindas R, Tvedt TH, Hatfield KJ, Reikvam $\mathrm{H}$, Bruserud $\mathrm{O}$. Preconditioning serum levels of endothelial cell-derived molecules and the risk of posttransplant complications in patients treated with allogeneic stem cell transplantation. J Transplant. 2014;2014:404096.

46. Biedermann BC, Tsakiris DA, Gregor M, Pober JS, Gratwohl A. Combining altered levels of effector transcripts in circulating $\mathrm{T}$ cells with a marker of endothelial injury is specific for active graft-versus-host disease. Bone Marrow Transplant. 2003;32(11): 1077-1084

47. Penack O, Henke E, Suh D, et al. Inhibition of neovascularization to simultaneously ameliorate graft-vs-host disease and decrease tumor growth. J Natl Cancer Inst. 2010;102(12):894-908.

48. Less JR, Skalak TC, Sevick EM, Jain RK. Microvascular architecture in a mammary carcinoma: branching patterns and vessel dimensions. Cancer Res. 1991;51(1):265273.

49. Huang Y, Goel S, Duda DG, Fukumura D, Jain RK. Vascular normalization as an emerging strategy to enhance cancer immunotherapy. Cancer Res. 2013;73(10): 2943-2948.

50. Tornavaca O, Chia M, Dufton N, et al. ZO1 controls endothelial adherens junctions, cell-cell tension, angiogenesis, and barrier formation. J Cell Biol. 2015;208(6):821-838.

51. Fanning AS, Little BP, Rahner C, Utepbergenov D, Walther Z, Anderson JM. The unique- 5 and -6 motifs of ZO- 1 regulate tight junction strand localization and scaffolding properties. Mol Biol Cell. 2007;18(3):721-731.

52. Umeda K, Ikenouchi J, Katahira-Tayama S, et al. ZO-1 and ZO-2 independently determine where claudins are polymerized in tight-junction strand formation. Cell. 2006;126(4):741-754

53. Reyat JS, Chimen M, Noy PJ, Szyroka J, Rainger GE, Tomlinson MG. ADAM10interacting tetraspanins Tspan5 and Tspan17 regulate VE-cadherin expression and promote $\mathrm{T}$ lymphocyte transmigration. J Immunol. 2017;199(2):666-676.

54. Vockel M, Vestweber D. How T cells trigger the dissociation of the endothelial receptor phosphatase VE-PTP from VE-cadherin. Blood. 2013;122(14):2512-2522.

55. Zhao Y, Ting KK, Li J, et al. Targeting vascular endothelial-cadherin in tumor-associated blood vessels promotes T-cell-mediated immunotherapy. Cancer Res. 2017;77 (16):4434-4447

56. Schulte D, Kuppers V, Dartsch N, et al. Stabilizing the VE-cadherin-catenin complex blocks leukocyte extravasation and vascular permeability. EMBO J. 2011;30 (20):4157-4170

57. McHale TM, Garciarena CD, Fagan RP, et 
al. Inhibition of vascular endothelial cell leak following Escherichia coli attachment in an experimental model of sepsis. Crit Care Med. 2018;46(8):e805-e810.

58. Han S, Lee SJ, Kim KE, et al. Amelioration of sepsis by TIE2 activation-induced vascular protection. Sci Transl Med. 2016;8(335):335ra355.

59. Wojciak-Stothard B, Tsang LY, Haworth SG. Rac and Rho play opposing roles in the regulation of hypoxia/reoxygenationinduced permeability changes in pulmonary artery endothelial cells. Am J Physiol Lung Cell Mol Physiol. 2005;288(4):L749-760.

60. Dias AT, Cintra AS, Frossard JC, et al.
Inhibition of phosphodiesterase 5 restores endothelial function in renovascular hypertension. J Transl Med. 2014;12:250

61. Chen W, Oberwinkler H, Werner F, et al. Atrial natriuretic peptide-mediated inhibition of microcirculatory endothelial $\mathrm{Ca} 2+$ and permeability response to histamine involves cGMP-dependent protein kinase I and TRPC6 channels. Arterioscler Thromb Vasc Biol. 2013;33(9):2121-2129.

62. Guilluy C, Sauzeau V, Rolli-Derkinderen $M$, et al. Inhibition of RhoA/Rho kinase pathway is involved in the beneficial effect of sildenafil on pulmonary hypertension. $\mathrm{Br}$ J Pharmacol. 2005;146(7):1010-1018.

63. Das A, Xi L, Kukreja RC.
Phosphodiesterase-5 inhibitor sildenafil preconditions adult cardiac myocytes against necrosis and apoptosis. Essential role of nitric oxide signaling. J Biol Chem. 2005;280(13):12944-12955.

64. Fukumoto S, Koyama H, Hosoi M, et al Distinct role of cAMP and cGMP in the cell cycle control of vascular smooth muscle cells: cGMP delays cell cycle transition through suppression of cyclin D1 and cyclin-dependent kinase 4 activation. Circ Res. 1999;85(11):985-991.

65.Zeiser R, von Bubnoff N, Butler J, et al Ruxolitinib for glucocorticoid-refractory acute graft-versus-host disease. N Engl J Med. 2020;382(19):1800-1810. 